\title{
A Possible Aoki Phase for Staggered Fermions
}

\author{
C. Aubin \\ Physics Dept., Columbia University, New York, NY 10027 \\ Qinghai Wang \\ Physics Dept., Washington University, St. Louis, MO 63130
}

\begin{abstract}
The phase diagram for staggered fermions is discussed in the context of the staggered chiral Lagrangian, extending previous work on the subject. When the discretization errors are significant, there may be an Aoki-like phase for staggered fermions, where the remnant $S O(4)$ taste symmetry is broken down to $S O(3)$. We solve explicitly for the mass spectrum in the 3 -flavor degenerate mass case and discuss qualitatively the $2+1$-flavor case. From numerical results we find that current simulations are outside the staggered Aoki phase. As for near-future simulations with more improved versions of the staggered action, it seems unlikely that these will be in the Aoki phase for any realistic value of the quark mass, although the evidence is not conclusive.

PACS numbers: 11.15.Ha,11.30.Qc,12.39.Fe
\end{abstract}




\section{INTRODUCTION}

Current simulations with dynamical staggered quarks have the benefits of both good chiral properties at finite lattice spacing and being computationally inexpensive [1]. The price for these features is that the quark doubling is not completely removed, and there are four "tastes" of quarks for each staggered flavor. Additionally, there are large $\mathcal{O}\left(a^{2}\right)$ scaling violations that arise and must be taken into account. Improved forms of the staggered action (e.g., "Asqtad" staggered quarks) reduce these scaling violations but they are still not negligible [2, 3, 4, 5, 6].

These scaling violations cause mass differences among the various tastes of light pseudoscalar mesons that result from the four quark tastes. In principle, these break the continuum $S U(4)$ taste symmetry down to the lattice subgroup [7], however, at $\mathcal{O}\left(a^{2}\right)$ there is a remnant $S O(4)$ taste symmetry which remains [8]. For each meson flavor, there are 15 tastes which fall into representations of $S O(4)$ : pseudoscalar (1), axial (4), tensor (6) and vector (4); there is also the $S U(4)$ singlet for a total of 16 . This approximate $S O(4)$ symmetry is broken down to the lattice subgroup at $\mathcal{O}\left(a^{4}\right)$. These splittings may give rise to a negative mass-squared of a meson, since there is no reason to assume the splittings are positive. This signals an instability of the vacuum, and thus a meson condensate may form.

The idea that there could be this sort of spontaneous symmetry breaking in the context of the flavor symmetry of Wilson fermions was first proposed by Aoki [9]. Lee and Sharpe showed that this could also occur for one flavor of staggered fermions as well [8]. In this case, they assumed one of these splittings to be negative and so the vector-taste pion condensed. All current formulations of staggered fermions find that these mass splittings are all positive, and thus this is not a likely scenario. In the case of multiple flavors, additional parameters which mix the axial and vector taste flavor-neutrals are negative in current simulations, so a possible "staggered-Aoki" phase may be more likely [10, 11] than originally thought. The phase structure of lattice theories has been studied for a variety of other lattice formulations recently [12, 13, 14, 15].

As we approach the broken phase, the quark mass $m$ becomes comparable to the discretization errors; in lattice units, $a m \sim a^{3} \Lambda_{\mathrm{QCD}}^{3}$. It is then that $m_{\text {meson }}^{2}$ may be negative and the $S O(4)$ taste symmetry is broken. The breaking is $S O(4)$ down to $S O(3)$, giving rise to three approximate Goldstone bosons. We expect a problem to arise in simulations if 
either very small quark masses or large lattice spacings are used. It seems as though current simulations are not in this region of the phase diagram, as we will discuss. Although it is not completely ruled out, it appears as though further improvements to the staggered action will also be safely away from the staggered-Aoki phase.

As pointed out in Ref. [8], we are not breaking an exact symmetry. The $S O(4)$ symmetry will be broken down to the lattice subgroup at higher order, so in this way it is unlike the Aoki phase for Wilson fermions, where the flavor symmetry is exact. Thus, the three Goldstone bosons actually have masses of $\mathcal{O}\left(a^{4}\right)$. Terms which arise at $\mathcal{O}\left(a^{4}\right)$ are subleading, however, and will only give rise to small corrections to our results here. For small enough lattice spacing they cannot change drastically the phase structure here.

The goal of this paper is to study the features of the particle spectrum in the broken phase as compared to the unbroken phase. We will do this first for the case where all three quark masses are degenerate, the 3-flavor case, which is simple and exactly solvable. We will then look empirically at the 2+1-flavor case, where the up and down quark masses are degenerate but different than the strange quark mass. This case is significantly more difficult than the 3-flavor case - we cannot analytically solve for the condensate in this case - and we will simply make some general comments on the critical point and how it relates to values of the parameters in current and future simulations. We will find that current simulations are not in the broken phase, and more improved versions of staggered fermions are likely not going to be in the broken phase, although we note that we cannot make exact statements about the more improved versions without performing simulations.

The approach we follow in this paper is similar to that of Refs. [8, 12, 13, 14, 15], using the chiral Lagrangian. This approach allows us to determine the mass spectrum of all the light pseudoscalar mesons rather simply. For the region we are discussing, $m \sim a^{2}$, the chiral expansion is quite natural, and we can use this to determine the meson masses with the dependence on all parameters explicit. We are not performing a complete analysis of the phase diagram, however. We will see that the phase diagram will turn out to be more complex than studied in this paper, but, although this will change certain features such as the mass spectrum, it will not change some central results of the analysis, as we will see.

This paper is structured as follows. In Sec. IIwe layout the necessary tools for the analysis: the staggered chiral Lagrangian including the $\mathcal{O}\left(a^{2}\right)$ taste-violating potential. Next, we determine the mass spectrum for the case of 3 degenerate flavors of quarks in Sec. III. In 
Sec. IV we discuss the 2+1-flavor case. Here we will not solve for the mass spectrum, but make some qualitative statements regarding this case. We will examine the features of the critical point with current simulations as a function of the quark masses in Sec. $\nabla$. Additionally we will look at how the point shifts with more improved staggered fermion formulations, and will see that indeed the problem becomes less severe in these cases. We finish up with some conclusions in Sec. VI]

\section{THE STAGGERED CHIRAL LAGRANGIAN}

The starting point of our analysis is the S $\chi$ PT Lagrangian for 3 flavors of quarks [10, 11]. The Lagrangian is written in terms of the field $\Sigma=\exp (i \Phi / f)$, a $12 \times 12$ matrix, with $\Phi$ given by:

$$
\Phi=\left(\begin{array}{ccc}
U & \pi^{+} & K^{+} \\
\pi^{-} & D & K^{0} \\
K^{-} & \bar{K}^{0} & S
\end{array}\right)
$$

where the elements shown are each $4 \times 4$ matrices, linear combinations of the Hermitian generators

$$
T_{a}=\left\{\xi_{5}, i \xi_{\mu 5}, i \xi_{\mu \nu}, \xi_{\mu}, \xi_{I}\right\}
$$

In other words, $U=\sum_{a} U_{a} T_{a}, K^{0}=\sum_{a} K_{a}^{0} T_{a}$, etc. In Euclidean space, the gamma matrices $\xi_{\mu}$ are Hermitian, and we use the notations $\xi_{\mu \nu} \equiv \xi_{\mu} \xi_{\nu}\left[\mu<\nu\right.$ in Eq. (2)], $\xi_{\mu 5} \equiv \xi_{\mu} \xi_{5}$ and $\xi_{I} \equiv I$ is the $4 \times 4$ identity matrix. Under the chiral $S U(12)_{L} \times S U(12)_{R}$ symmetry, $\Sigma \rightarrow L \Sigma R^{\dagger}$. The components of the diagonal (flavor-neutral) elements $\left(U_{a}, D_{a}, S_{a}\right)$ are real; while the other (flavor-charged) fields are complex $\left(\pi_{a}^{+}, K_{a}^{0}\right.$, etc.), such that $\Phi$ is Hermitian.

The Lagrangian is given by

$$
\mathcal{L}=\frac{f^{2}}{8} \operatorname{Tr}\left(\partial_{\mu} \Sigma \partial_{\mu} \Sigma^{\dagger}\right)-\frac{1}{4} \mu f^{2} \operatorname{Tr}\left(\mathcal{M} \Sigma+\mathcal{M} \Sigma^{\dagger}\right)+\frac{2 m_{0}^{2}}{3}\left(U_{I}+D_{I}+S_{I}\right)^{2}+a^{2} \mathcal{V}
$$

where $\mu$ is a constant with dimensions of mass, $f$ is the tree-level pion decay constant, the $m_{0}^{2}$ term includes the 3 flavor-neutral taste-singlet fields $\left(m_{0} \rightarrow \infty\right.$ at the end to decouple 
the taste-singlet $\eta_{I}^{\prime}$, and $\mathcal{V}=\mathcal{U}+\mathcal{U}^{\prime}$ is the taste-symmetry breaking potential given by

$$
\begin{aligned}
-\mathcal{U}= & C_{1} \operatorname{Tr}\left(\xi_{5}^{(3)} \Sigma \xi_{5}^{(3)} \Sigma^{\dagger}\right) \\
& +C_{3} \frac{1}{2} \sum_{\nu}\left[\operatorname{Tr}\left(\xi_{\nu}^{(3)} \Sigma \xi_{\nu}^{(3)} \Sigma\right)+h . c .\right] \\
& +C_{4} \frac{1}{2} \sum_{\nu}\left[\operatorname{Tr}\left(\xi_{\nu 5}^{(3)} \Sigma \xi_{5 \nu}^{(3)} \Sigma\right)+h . c .\right] \\
& +C_{6} \sum_{\mu<\nu} \operatorname{Tr}\left(\xi_{\mu \nu}^{(3)} \Sigma \xi_{\nu \mu}^{(3)} \Sigma^{\dagger}\right) \\
-\mathcal{U}^{\prime}= & C_{2 V} \frac{1}{4} \sum_{\nu}\left[\operatorname{Tr}\left(\xi_{\nu}^{(3)} \Sigma\right) \operatorname{Tr}\left(\xi_{\nu}^{(3)} \Sigma\right)+h . c .\right] \\
& +C_{2 A} \frac{1}{4} \sum_{\nu}\left[\operatorname{Tr}\left(\xi_{\nu 5}^{(3)} \Sigma\right) \operatorname{Tr}\left(\xi_{5 \nu}^{(3)} \Sigma\right)+h . c .\right] \\
& +C_{5 V} \frac{1}{2} \sum_{\nu}\left[\operatorname{Tr}\left(\xi_{\nu}^{(3)} \Sigma\right) \operatorname{Tr}\left(\xi_{\nu}^{(3)} \Sigma^{\dagger}\right)\right] \\
& +C_{5 A} \frac{1}{2} \sum_{\nu}\left[\operatorname{Tr}\left(\xi_{\nu 5}^{(3)} \Sigma\right) \operatorname{Tr}\left(\xi_{5 \nu}^{(3)} \Sigma^{\dagger}\right)\right] .
\end{aligned}
$$

The $\xi_{B}^{(3)}$ in $\mathcal{V}$ are block-diagonal $12 \times 12$ matrices

$$
\xi_{B}^{(3)}=\left(\begin{array}{ccc}
\xi_{B} & 0 & 0 \\
0 & \xi_{B} & 0 \\
0 & 0 & \xi_{B}
\end{array}\right),
$$

with $B \in\{5, \mu 5, \mu \nu(\mu<\nu), \mu, I\}$.

The mass matrix, $\mathcal{M}$, is the $12 \times 12$ matrix

$$
\mathcal{M}=\left(\begin{array}{ccc}
m_{u} I & 0 & 0 \\
0 & m_{d} I & 0 \\
0 & 0 & m_{s} I
\end{array}\right)
$$

We will in later sections take either all three quark masses to be degenerate or $m_{u}=m_{d} \neq$ $m_{s}$.

As noted in Refs. 8, 11], this potential, although breaking the taste symmetry at $\mathcal{O}\left(a^{2}\right)$, has an accidental $S O(4)$ symmetry. This implies a degeneracy in the masses among different tastes of a given flavor meson, which is seen in the tree-level masses of the pseudoscalar mesons. We can classify these mesons into irreducible representations of $S O(4)$. The mass for the meson $M$ (composed of quarks $a$ and $b$ ) with taste $B$, is given at tree-level by

$$
m_{M_{B}}^{2}=\mu\left(m_{a}+m_{b}\right)+a^{2} \Delta_{B}
$$


with

$$
\begin{aligned}
\Delta\left(\xi_{5}\right) & \equiv \Delta_{P}=0 \\
\Delta\left(\xi_{\mu 5}\right) & \equiv \Delta_{A}=\frac{16}{f^{2}}\left(C_{1}+3 C_{3}+C_{4}+3 C_{6}\right) \\
\Delta\left(\xi_{\mu \nu}\right) & \equiv \Delta_{T}=\frac{16}{f^{2}}\left(2 C_{3}+2 C_{4}+4 C_{6}\right) \\
\Delta\left(\xi_{\mu}\right) & \equiv \Delta_{V}=\frac{16}{f^{2}}\left(C_{1}+C_{3}+3 C_{4}+3 C_{6}\right) \\
\Delta\left(\xi_{I}\right) & \equiv \Delta_{I}=\frac{16}{f^{2}}\left(4 C_{3}+4 C_{4}\right) .
\end{aligned}
$$

As in Ref. [11], we do not include the $m_{0}^{2}$ terms or the terms from $\mathcal{U}^{\prime}$ in these masses. Those terms only affect the flavor-neutral mesons and allow for mixings in the basis of Eq. (II), the "flavor basis." We can expand $\mathcal{U}^{\prime}$ to quadratic order to find these mixings take the same form as the singlet term in the Lagrangian:

$$
\begin{array}{cr}
+\frac{a^{2} \delta_{V}^{\prime}}{2}\left(U_{\mu}+D_{\mu}+S_{\mu}\right)^{2} & \text { taste }- \text { vector } \\
+\frac{a^{2} \delta_{A}^{\prime}}{2}\left(U_{\mu 5}+D_{\mu 5}+S_{\mu 5}\right)^{2} & \text { taste }- \text { axial }
\end{array}
$$

where we have defined the parameters

$$
\delta_{V(A)}^{\prime} \equiv \frac{16}{f^{2}}\left[C_{2 V(A)}-C_{5 V(A)}\right]
$$

This requires us to resum the flavor-neutral propagators in the three cases: taste-vector, taste-axial and taste-singlet [11]. Performing this resummation is equivalent to writing everything in terms of the "physical basis" of our mesons, the $\pi^{0}, \eta$ and $\eta^{\prime}$ for each of the three tastes here, which are the true eigenstates of the mass matrix. These are the additional parameters discussed in the introduction which may cause the vector or axial $\eta^{\prime}$ mass-squared to be negative, thereby introducing a staggered Aoki phase similar to that discussed in Ref. [8].

The mass eigenstates of the flavor-neutral mass matrix, for non-degenerate flavors, are quite complicated, but the completely general case is not needed. The two cases relevant to current simulations are the fully degenerate case $\left(m_{u}=m_{d}=m_{s} \equiv m\right)$, which we refer to as the "3-flavor" case, and the case where two flavors are degenerate $\left(m_{u}=m_{d} \equiv m \neq m_{s}\right)$, or the "2+1" case. We will examine the 3-flavor case in Sec. III and discuss the 2+1-flavor case in Sec. IV] 


\section{MASS SPECTRUM IN THE BROKEN PHASE FOR 3 DEGENERATE FLA- VORS}

We first discuss the simplest case, where all three quarks are degenerate in mass, $m_{u}=m_{d}=m_{s} \equiv m$. First we need the eigenvalues of the full flavor-neutral mass matrix. The vector, axial and singlet tastes are the only tastes which mix and therefore require rediagonalizing the mass matrix. The singlet-taste masses do not involve the new parameters given in Eq. (12), and so we will not focus on their masses. Also, for the singlet, we take $m_{0} \rightarrow \infty$ on theoretical grounds, but there is no similar reasoning to dictate the values of $\delta_{V}^{\prime}$ or $\delta_{A}^{\prime}$; they must be determined from fits to lattice data [16, 17].

For the 3-flavor case, the mass eigenvalues for the vector (axial) tastes are given by

$$
\begin{aligned}
& m_{\pi_{V(A)}^{0}}^{2}=m_{\eta_{V(A)}}^{2}=2 \mu m+a^{2} \Delta_{V(A)} \\
& m_{\eta_{V(A)}^{\prime}}^{2}=2 \mu m+a^{2} \Delta_{(V) A}+\frac{3}{4} a^{2} \delta_{V(A)}^{\prime} .
\end{aligned}
$$

We have taken into account the transition from four to one tastes per flavor with the additional factor of $1 / 4$ in the $a^{2} \delta_{V(A)}^{\prime}$ term both in Eq. (13) and below. More discussion on how to account for this transition can be found in Refs. [11, 18, 19].

Lee and Sharpe's original discussion in the one-flavor case focused on the possibility that one of the splittings in Eq. (91) may be negative and cause a meson mass-squared to go negative for certain values of the quark masses. This signals a vacuum instability and the formation of a meson condensate. As noted in Ref. [8], all current simulations with staggered quarks show that $\Delta_{B}>0$ for all $B[\underline{5}, 6]$. On the other hand, as is clear from Eq. (13), there is another possibility for a meson mass-squared to become negative, when one of the "hairpin" parameters, $\delta_{V(A)}^{\prime}$, is negative. ${ }^{1}$ In current fits to MILC simulations, both $\delta_{V}^{\prime}$ and $\delta_{A}^{\prime}$ are negative [16, 17], so we can see it is more probable to encounter an Aoki-like phase with staggered fermions in this way. Current simulations are outside the broken phase [5, 6]. However, there is the question of how close current simulations are to this broken phase and how improving the action more changes this.

\footnotetext{
1 The term "hairpin" comes from the fact that these two-point interactions are disconnected at the quark level. See Refs. [1, 19] for details.
} 
It is straightforward to see that the vector (axial) $\eta^{\prime}$ mass-squared is negative if

$$
a^{2} \delta_{V(A)}^{\prime}<a^{2} \delta_{V(A), \text { crit }}^{\prime}=-\frac{4}{3}\left[2 \mu m+a^{2} \Delta_{V(A)}\right] .
$$

$\Delta_{A}<\Delta_{V}$ in simulations, so Eq. (14) implies that $\left|\delta_{A, \text { crit }}^{\prime}\right|<\left|\delta_{V \text {,crit }}^{\prime}\right|$. Thus, $\delta_{A}^{\prime}$ is more likely to be less than $\delta_{A, \text { crit }}^{\prime}$ than for the vector taste. We will assume for the following analysis that this is the case, although the analysis for the vector mesons would be analogous.

To find the vacuum state of the theory, we must minimize the potential

$$
\mathcal{W}=-\frac{1}{4} \mu m f^{2} \operatorname{Tr}\left(\Sigma+\Sigma^{\dagger}\right)+a^{2} \mathcal{U}+a^{2} \mathcal{U}^{\prime}
$$

where we have substituted $\mathcal{M}=\operatorname{diag}(m I, m I, m I)$.

This calculation is most simply done in the physical basis, where everything is written in terms of $\pi^{0}, \eta$ and $\eta^{\prime}$ instead of the flavor-basis mesons $U, D$ and $S$. In the 3-flavor case, these two bases are related by

$$
\begin{aligned}
& \pi_{B}^{0}=\frac{1}{\sqrt{2}}\left(U_{B}-D_{B}\right), \\
& \eta_{B}=\frac{1}{\sqrt{6}}\left(U_{B}+D_{B}-2 S_{B}\right), \\
& \eta_{B}^{\prime}=\frac{1}{\sqrt{3}}\left(U_{B}+D_{B}+S_{B}\right),
\end{aligned}
$$

with $B$ any of the 16 tastes. In the $2+1$-flavor case, or any case where some masses are non-degenerate, this relationship does not hold for all tastes. ${ }^{2}$ For the taste singlets, Eq. (16) is valid in the $m_{0} \rightarrow \infty$ limit for any quark masses. For the tensor and pseudoscalar tastes, this also holds. ${ }^{3}$ For the vector and axial tastes, however, the relationship is much more complicated in the non-degenerate case, although we will not need the explicit relationship in that case.

Since we are assuming that the axial $\eta^{\prime}$ mass-squared is going negative, we are going to keep only this meson in $\Phi$. This is valid right near the critical point and we assert that this remains valid so long as no other mass-squared is negative in the new phase. If this occurs,

\footnotetext{
${ }^{2}$ We remark that often in continuum $\chi \mathrm{PT}$ this relationship is used between the two bases. In that case, when $m_{u} \neq m_{d}$, there are additional mass-mixing terms between the $\pi^{0}$ and the $\eta$ which are usually neglected.

${ }^{3}$ We note that we need not write the tensor and pseudoscalar taste mesons in the physical basis, since they do not mix. Since this is an orthogonal transformation, the bases are equivalent. Writing them in the physical basis makes the subsequent discussion simpler.
} 
then there will be a more complicated pattern of symmetry breaking. We will see that this is a possibility, and thus the phase diagram is richer than discussed here. However, we will only focus on one region of the phase diagram here.

Keeping only the $\eta_{A}^{\prime}, \Phi$ and $\Sigma$ are block-diagonal in flavor space. We can write the condensate $\langle\Sigma\rangle$ in terms of the five real numbers $A$ and $B_{\mu}$ :

$$
\langle\Sigma\rangle=A\left(1_{12 \times 12}\right)+i B_{\mu}\left(i \xi_{\mu 5}^{(3)}\right)
$$

with the condition that $A^{2}+B_{\mu} B_{\mu}=1$. We write $A=\cos \theta$ and substitute this into $\mathcal{W}$ to get (dropping constants)

$$
\mathcal{W}=-3 f^{2}\left[2 \mu m \cos \theta+\frac{1}{2}\left(a^{2} \Delta_{A}+\frac{3}{4} a^{2} \delta_{A}^{\prime}\right) \cos ^{2} \theta\right]
$$

To find the condensate, we wish to minimize this potential. If $\delta_{A}^{\prime}$ is greater than $\delta_{A, \text { crit }}^{\prime} \equiv \delta_{c}^{\prime}$, then the minimum always lies at $\theta=0$ if $\mu m>0$ or $\theta=\pi$ for $\mu m<0$. In this phase, the unbroken phase, $\langle\Sigma\rangle=\operatorname{sign}(\mu m) 1_{12 \times 12}$ and the mass spectrum is exactly that discussed above (for $\mu m<0$ make the replacement $\mu m \rightarrow|\mu m|[8]$ ).

The more interesting case is when we take $\delta_{A}^{\prime}<\delta_{c}^{\prime}$. Since $\delta_{A}^{\prime}$ is negative in this region, we write $\delta_{A}^{\prime}=-\left|\delta_{A}^{\prime}\right|$. Here, we find the potential is minimized when

$$
\cos \theta=\frac{8 \mu m}{3 a^{2}\left|\delta_{A}^{\prime}\right|-4 a^{2} \Delta_{A}}
$$

so the condensate has the form

$$
\langle\Sigma\rangle=\left(\begin{array}{ccc}
\exp \left[i \theta n_{\mu}\left(i \xi_{\mu 5}\right)\right] & 0 & 0 \\
0 & \exp \left[i \theta n_{\mu}\left(i \xi_{\mu 5}\right)\right] & 0 \\
0 & 0 & \exp \left[i \theta n_{\mu}\left(i \xi_{\mu 5}\right)\right]
\end{array}\right)
$$

where $n_{\mu}$ is a unit vector.

The condensate breaks the $S O(4)$ symmetry down to $S O(3)$, giving us three Goldstone bosons. As noted in the introduction, these are not exactly Goldstone bosons, because we are not breaking an exact symmetry. The $S O(4)$ symmetry will be broken down to the lattice subgroup by terms of $\mathcal{O}\left(a^{4}\right)$. Thus the masses of these pseudo-Goldstone Bosons are $m_{\mathrm{GB}}^{2} \sim a^{4} \Lambda_{\mathrm{QCD}}^{6}$

Since the condensate is proportional to the identity in flavor space, the flavor-charged mesons (the $\pi^{+}, K^{0}$, etc.) have the same form as the $\pi^{0}$ and $\eta$ in the broken phase. 
Thus, below we only list the masses for the $\pi^{0}$ of the various tastes, but keep in mind that $m_{\pi^{0}}^{2}=m_{\pi^{+}}^{2}=m_{\eta}^{2}=m_{K^{+}}^{2}=m_{K^{0}}^{2}$ for all tastes. Additionally, this form of the condensate implies that there is no flavor mixing between the $\pi^{0}, \eta$ and $\eta^{\prime}$ in this case. We pick the 4-direction for the condensate, so that $n_{\mu}=\delta_{4 \mu}$ to get explicit results for the masses. We also define the quantities

$$
\begin{aligned}
& \delta_{2 A}^{\prime}=\frac{16}{f^{2}} C_{2 A}, \\
& \delta_{5 A}^{\prime}=\frac{16}{f^{2}} C_{5 A},
\end{aligned}
$$

so $\delta_{A}^{\prime}=\delta_{2 A}^{\prime}-\delta_{5 A}^{\prime}$, and similarly for $A \rightarrow V$. We will organize the masses according to taste, by whether or not they mix.

\section{A. Tastes which mix}

The $\xi_{4} \& \xi_{5}$ tastes mix with each other, as do the $\xi_{i 4} \& \xi_{i 5}$ tastes (with $i=1,2,3$ ). For the $\xi_{4} \& \xi_{5}$ tastes, we have the same mixing for any of the mesons. With $\phi$ being $\pi^{+}, \pi^{0}$, $K^{+}, K^{0}, \eta$, or $\eta^{\prime}$, we define the new fields

$$
\begin{aligned}
& \phi_{4}^{\text {new }}=\cos \theta \phi_{4}-\sin \theta \phi_{5} \\
& \phi_{5}^{\text {new }}=\sin \theta \phi_{4}+\cos \theta \phi_{5} .
\end{aligned}
$$

These new fields are defined so that at $\cos \theta=1$, the critical point, $\phi_{4}^{\text {new }}$ is purely $\phi_{4}$ and similarly $\phi_{5}^{\text {new }}=\phi_{5}$. This means that in the chiral limit, at $\cos \theta=0$, this is reversed: $\phi_{4}^{\text {new }}$ is purely $\phi_{5}$ and vice versa. In terms of these new mass eigenstates, we find the masses

$$
\begin{aligned}
& m_{\pi_{4}^{0, \text { new }}}^{2}=a^{2} \Delta_{V}-a^{2} \Delta_{A}+\frac{3}{4} a^{2}\left|\delta_{A}^{\prime}\right| \\
& m_{\pi_{5}^{0, \text { new }}}^{2}=m_{\eta_{5}^{\prime \text { new }}}^{2}=-a^{2} \Delta_{A}+\frac{3}{4} a^{2}\left|\delta_{A}^{\prime}\right| \\
& m_{\eta_{4}^{\prime} \text { new }}^{2}=a^{2} \Delta_{V}-a^{2} \Delta_{A}+\frac{3}{4} a^{2}\left|\delta_{A}^{\prime}\right|-\frac{3}{4} a^{2} \delta_{V}^{\prime}
\end{aligned}
$$

Note the combination $-4 a^{2} \Delta_{A}+3 a^{2}\left|\delta_{A}^{\prime}\right|$, which appears frequently, is positive, so Eqs. (24) through (26) are all positive in the broken phase.

As for the $\xi_{i 4} \& \xi_{i 5}$ tastes, we have the same mixing as in Eq. (22), with $4 \rightarrow i 4$ and 
$5 \rightarrow i 5$, again with $\phi$ referring to any of the six mesons. These have the masses

$$
\begin{aligned}
& m_{\pi_{i 4}^{0 \text { new }}}^{2}=m_{\eta_{i^{\prime}}^{\prime \text { new }}}^{2}=a^{2} \Delta_{T}-a^{2} \Delta_{A}+\frac{3}{4} a^{2}\left|\delta_{A}^{\prime}\right| \\
& m_{\pi_{i 5}^{0, \text { new }}}^{2}=\frac{3}{4} a^{2}\left|\delta_{A}^{\prime}\right| \\
& m_{\eta_{i 5}^{\prime} \text { new }}^{2}=0 .
\end{aligned}
$$

Again, all of these squared masses are positive for our range of parameter values. We see that the $\eta_{i 5}^{\prime \text { new }}$ are the three expected Goldstone bosons.

\section{B. Tastes which do not mix}

The rest of the tastes do not mix with each other, and so we just list the masses for each of these states.

$$
\begin{aligned}
m_{\pi_{I}^{0}}^{2}= & a^{2} \Delta_{V}-a^{2} \Delta_{A}+\frac{3}{4} a^{2}\left|\delta_{A}^{\prime}\right|+\frac{64 m^{2} \mu^{2}\left(a^{2} \Delta_{I}-a^{2} \Delta_{V}\right)}{\left(4 a^{2} \Delta_{A}-3 a^{2}\left|\delta_{A}^{\prime}\right|\right)^{2}} \\
m_{\pi_{45}^{0}}^{2}= & -a^{2} \Delta_{A}+\frac{3}{4} a^{2}\left|\delta_{A}^{\prime}\right|+\frac{64 m^{2} \mu^{2} a^{2} \Delta_{A}}{\left(4 a^{2} \Delta_{A}-3 a^{2}\left|\delta_{A}^{\prime}\right|\right)^{2}} \\
m_{\eta_{45}^{\prime}}^{2}= & -a^{2} \Delta_{A}+\frac{3}{4} a^{2}\left|\delta_{A}^{\prime}\right|+\frac{16 m^{2} \mu^{2}}{4 a^{2} \Delta_{A}-3 a^{2}\left|\delta_{A}^{\prime}\right|} \\
m_{\pi_{i}^{0}}^{2}= & a^{2} \Delta_{T}-a^{2} \Delta_{A}+\frac{3}{4} a^{2}\left|\delta_{A}^{\prime}\right|+\frac{64 m^{2} \mu^{2}\left(a^{2} \Delta_{V}-a^{2} \Delta_{T}\right)}{\left(4 a^{2} \Delta_{A}-3 a^{2}\left|\delta_{A}^{\prime}\right|\right)^{2}} \\
m_{\eta_{i}^{\prime}}^{2}= & a^{2} \Delta_{T}-a^{2} \Delta_{A}+\frac{3}{4} a^{2}\left|\delta_{A}^{\prime}\right|+\frac{16 m^{2} \mu^{2}\left(4 a^{2} \Delta_{V}-4 a^{2} \Delta_{T}+3 a^{2} \delta_{V}^{\prime}\right)}{\left(4 a^{2} \Delta_{A}-3 a^{2}\left|\delta_{A}^{\prime}\right|\right)^{2}} \\
m_{\pi_{i j}^{0}}^{2}= & \frac{3}{4} a^{2}\left|\delta_{A}^{\prime}\right|+\frac{64 m^{2} \mu^{2}\left(a^{2} \Delta_{T}-a^{2} \Delta_{A}\right)}{\left(4 a^{2} \Delta_{A}-3 a^{2}\left|\delta_{A}^{\prime}\right|\right)^{2}} \\
m_{\eta_{i j}^{\prime}}^{2}= & \frac{3}{4} a^{2}\left|\delta_{A}^{\prime}\right|-\frac{3}{4} a^{2} \delta_{2 V}^{\prime}-\frac{3}{4} a^{2} \delta_{5 V}^{\prime} \\
& +\frac{16 m^{2} \mu^{2}\left(4 a^{2} \Delta_{T}-4 a^{2} \Delta_{A}+3 a^{2} \delta_{2 V}^{\prime}+3 a^{2} \delta_{5 V}^{\prime}\right)}{\left(4 a^{2} \Delta_{A}-3 a^{2}\left|\delta_{A}^{\prime}\right|\right)^{2}} .
\end{aligned}
$$

In the chiral limit, when $m=0$, we have several degeneracies, similar to those found in Ref. 8]. In this limit, the states which mix are purely one state (e.g., $\pi_{i 4}^{0, \text { new }}=\pi_{i 5}^{0}$ ), and we denote these by the pure state. When $m=0$, the $U(1)_{A}$ is restored, and we see that $m_{\pi_{i 5}^{0}}^{2}=m_{\pi_{i}^{0}}^{2}=m_{\eta_{i}^{\prime}}^{2}, m_{\pi_{4}^{0}}^{2}=m_{\pi_{45}^{0}}^{2}=m_{\eta_{45}^{\prime}}^{2}$ and $m_{\pi_{5}^{0}}^{2}=m_{\pi_{I}^{0}}^{2}$. Additionally, we have $m_{\pi_{i 4}^{0}}^{2}=m_{\pi_{i j}^{0}}^{2}$, which occurs in the massless case due to an accidental $S O(3)$ symmetry about the minimum of the potential when $\delta_{A}^{\prime}=0$. This degeneracy recurs at the critical point for a different reason: due to the restoration of the $S O(4)$ symmetry. Note that the limits of $m \rightarrow 0$ and 
$\delta_{A}^{\prime} \rightarrow \delta_{c}^{\prime}$ do not commute. If we take $\delta_{A}^{\prime} \rightarrow \delta_{c}^{\prime}$ first, the masses all become those of the unbroken phase, so taking $m \rightarrow 0$ gives the massless forms of Eq. (8). However, taking $m \rightarrow 0$ first while in the broken phase then the $\delta_{A}^{\prime} \rightarrow \delta_{c}^{\prime}$ limit results in a much different spectrum which is not the same as in the unbroken phase.

The appearance of the "incomplete" hairpin parameters $\delta_{2 V}^{\prime}$ and $\delta_{5 V}^{\prime}$ in the $\eta_{i j}^{\prime}$ mass shows that the phase structure is more complicated than just the two phases we have discussed. Since we do not know the size or the sign of either of these two parameters, these could cause the mass-squared of the $\eta_{i j}^{\prime}$ to go negative. If this is the case, the spectrum we have laid out will not be strictly valid, and we would have to take into account the possibility of a condensate also in the $i j$-directions.

We can determine the requirements on the parameters to cause the $\eta_{i j}^{\prime}$ mass-squared to be negative. This happens when

$$
a^{2} \delta_{5 V}^{\prime}+a^{2} \delta_{2 V}^{\prime}>\frac{3 a^{2}\left|\delta_{A}^{\prime}\right|\left(3 a^{2}\left|\delta_{A}^{\prime}\right|-4 a^{2} \Delta_{A}\right)^{2}+256\left(a^{2} \Delta_{T}-a^{2} \Delta_{A}\right) \mu^{2} m^{2}}{3\left(3 a^{2}\left|\delta_{A}^{\prime}\right|-4 a^{2} \Delta_{A}\right)^{2}-192 \mu^{2} m^{2}} .
$$

So in the massless case (where we will see later that we are most likely to be in the broken phase), this condition requires $a^{2} \delta_{5 V}^{\prime}+a^{2} \delta_{2 V}^{\prime}>a^{2}\left|\delta_{A}^{\prime}\right|$. So the further we go into the broken phase, $\left|\delta_{A}^{\prime}\right|$ gets larger, meaning that we have a less chance of finding $m_{\eta_{i j}^{\prime}}^{2}<0$. Here we are studying the simplest case, where this criterion is not met, and the spectrum above is valid.

We cannot know the values of these parameters from first principles; we must calculate them from fits to lattice data. This can be done by calculating quantities for non-Goldstone mesons (such as the taste-singlet or taste-vector charged pions). The specific combination $\delta_{A}^{\prime}=\delta_{2 A}^{\prime}-\delta_{5 A}^{\prime}$ (and similarly for $A \rightarrow V$ ) appears when calculating the pseudoscalar taste masses and decay constants, but in other taste mesons, we find that $\delta_{2 A}^{\prime}+\delta_{5 A}^{\prime}$ also appears. In order to have a complete determination of the taste-violating parameters, one must calculate the expressions for the masses and decay constants for each of the other tastes and match those expressions to lattice simulation data.

This will not change our results in Sec. $\nabla$ however, since those results are focused on studying the location of the critical point, $\delta_{c}^{\prime}$. The criterion $\delta_{A}^{\prime}>\delta_{c}^{\prime}$ is required for any symmetry breaking; if this does not hold we have the mass spectrum of the unbroken phase. If Eq. (37) holds, this would change the spectrum of masses and the breaking pattern as well as the condensate itself. We will discuss the functional dependence of $\delta_{c}^{\prime}$ on the various parameters, and examine the possibility of current (and near future) simulations 
being performed in the broken phase. If we are in the broken phase, then we would have to determine if Eq. (37) holds; if not, the spectrum discussed above would be correct. If it does, a more complete study should be done of the resulting mass spectrum. As it is, a very rich phase structure is possible for staggered fermions.

\section{THE BROKEN PHASE FOR $2+1$ FLAVORS}

In the $2+1$ case, we set $m_{u}=m_{d} \equiv m_{l} \neq m_{s}$, so $\mathcal{M}=\operatorname{diag}\left(m_{l} I, m_{l} I, m_{s} I\right)$. Here when diagonalizing the flavor neutral axial taste mass matrix, the mass eigenstates for the axial tastes to be given by [11]

$$
\begin{aligned}
m_{\pi_{A}^{0}}^{2} & =2 \mu m_{l}+a^{2} \Delta_{A} \\
m_{\eta_{A}}^{2} & =\frac{1}{2}\left[2 \mu\left(m_{l}+m_{s}\right)+2 a^{2} \Delta_{A} \frac{3}{4} a^{2} \delta_{A}^{\prime}+Z\right] \\
m_{\eta_{A}^{\prime}}^{2} & =\frac{1}{2}\left[2 \mu\left(m_{l}+m_{s}\right)+2 a^{2} \Delta_{A}+\frac{3}{4} a^{2} \delta_{A}^{\prime}-Z\right] ; \\
Z & \equiv \sqrt{4 \mu^{2}\left(m_{s}-m_{l}\right)^{2}-a^{2} \delta_{A}^{\prime} \mu\left(m_{s}-m_{l}\right)+\frac{9\left(a^{2} \delta_{A}^{\prime}\right)^{2}}{16}} .
\end{aligned}
$$

The vector tastes have the same form (with $V \rightarrow A$ ), but again we are only focusing on the axial taste masses vanishing.

Our definitions of $\eta_{A}$ and $\eta_{A}^{\prime}$ are different from that in Ref. [11]. We define the $\eta_{A}$ as the field which becomes degenerate with the $\pi_{A}^{0}$ when $m_{l}=m_{s}$. Thus the $\eta_{A}$ from Ref. 11] is

now our $\eta_{A}^{\prime}$ since $\delta_{A}^{\prime}<0$ (as determined from simulations). Using this definition, it is the $\eta_{A}^{\prime}$ mass which vanishes at the critical value of $\delta_{A}^{\prime}$, just as in the 3 -flavor case. In the $2+1$-flavor case, this critical point is given by the expression

$$
a^{2} \delta_{c}^{\prime}=-\frac{4\left(2 \mu m_{l}+a^{2} \Delta_{A}\right)\left(2 \mu m_{s}+a^{2} \Delta_{A}\right)}{2 \mu\left(m_{l}+2 m_{s}\right)+3 a^{2} \Delta_{A}} .
$$

The condensate has the form

$$
\langle\Sigma\rangle=\left(\begin{array}{ccc}
\exp \left[i \theta n_{\mu}\left(i \xi_{\mu 5}\right)\right] & 0 & 0 \\
0 & \exp \left[i \theta n_{\mu}\left(i \xi_{\mu 5}\right)\right] & 0 \\
0 & 0 & \exp \left[i \kappa \theta n_{\mu}\left(i \xi_{\mu 5}\right)\right]
\end{array}\right)
$$

$n_{\mu}$ is a unit vector in the $\mu$ direction and we have defined

$$
\kappa=\frac{8 \mu\left(m_{s}-m_{l}\right)-a^{2} \delta_{A}^{\prime}-4 Z}{2 a^{2} \delta_{A}^{\prime}},
$$


which arises from the relationship between the $\eta_{A}^{\prime}$ and the flavor basis

$$
\eta_{A}^{\prime} \equiv \mathcal{N}\left(U_{A}+D_{A}+\kappa S_{A}\right)
$$

with $\mathcal{N}$ a normalization constant. Note that in the limit that $\delta_{A}^{\prime} \rightarrow-\infty$, or $m_{s} \rightarrow m_{l}$, $\kappa \rightarrow 1$, giving us the same definition of the $\eta_{A}^{\prime}$ as in the 3 -flavor case.

$\theta$ in Eq. (41) is the solution which minimizes the potential, which in this case has the form

$$
\begin{aligned}
\mathcal{W}= & -f^{2}\left[4 \mu m_{l} \cos (\theta)+2 \mu m_{s} \cos (\kappa \theta)+\left(a^{2} \Delta_{A}+\frac{1}{2} a^{2} \delta_{A}^{\prime}\right) \cos ^{2}(\theta)\right. \\
& \left.+\frac{1}{2}\left(a^{2} \Delta_{A}+\frac{1}{4} a^{2} \delta_{A}^{\prime}\right) \cos ^{2}(\kappa \theta)-\frac{1}{2} a^{2} \delta_{A}^{\prime} \sin (\theta) \sin (\kappa \theta)\right]+ \text { const. }
\end{aligned}
$$

We plot this potential in Fig. 1 for the unbroken phase (so that $\delta_{A}^{\prime}>\delta_{c}^{\prime}$ ). In this and subsequent figures, we are plotting the dimensionless quantity $W=a^{2} \mathcal{W} / f^{2}$ as a function of $\theta$. There are two minima, one of which is the global minimum at $\theta=0$, which defines the vacuum state as we expect in the unbroken phase. At the critical point, $\delta_{A}^{\prime}=\delta_{c}^{\prime}$, shown in Fig. 2, both minima have flattened out; if we were to expand Eq. (44) about $\theta=0$, we would see the $\theta^{2}$ term vanish here. Once we have entered the broken phase, in Fig. 3, the minima have both shifted towards each other, and the global minimum is at a non-zero value of $\theta$. As $\delta_{A}^{\prime} \rightarrow-\infty$, we find that the minima correspond to two equivalent solutions for the ground state here. In terms of the potential and the critical point, the $\delta_{A}^{\prime} \rightarrow-\infty$ limit is similar to the chiral limit [see Eq. (40)], where the $U(1)_{A}$ symmetry is restored. In the chiral limit, there are two minima given by $\theta=0$ or $\pi$, and they are equivalent. Shifting $\theta$ by $\pi$ merely sends $\Sigma \rightarrow-\Sigma$, which is a symmetry of the Lagrangian in the massless case. In the case of $\delta_{A}^{\prime} \rightarrow-\infty$, the minima are not at 0 or $\pi$, but roughly near $\pi / 2$ and $3 \pi / 2$, and are equivalent minima in the potential.

We cannot solve for an analytic solution for $\cos \theta$ in this case as we did in the 3-flavor case, although we can solve for the masses of the mesons in the broken phase in terms of $\theta$, or more appropriately $\cos (\theta)$ and $\cos (\kappa \theta)$. As in the 3 -flavor case, there is taste mixing, but now the condensate is not proportional to the identity in flavor space. This implies that we should find mixing between the $\eta$ and $\eta^{\prime}$ mesons. Since the condensate is still diagonal in flavor space, there will be no mixing between the charged fields. The up and down quarks are degenerate, so the $\pi^{0}, \pi^{+}$and $\pi^{-}$have the same general form; there are only mixings 
among the tastes but there is no flavor mixing. The expressions are complicated and not very enlightening, so instead of looking directly at the mass spectrum, we will study this phase numerically. In the next section, we will look at the critical point as a function of the various parameters and see the criteria for entering this staggered Aoki phase.

\section{NUMERICAL RESULTS}

With current MILC simulations, we now discuss the dependence of the critical point on the various parameters in our theory. We will look at data from the "coarse" $(a \approx 0.125 \mathrm{fm})$ and "fine" ( $a \approx 0.09 \mathrm{fm})$ MILC lattices $[5,6]$. We will look first at the quark mass dependence on the two different lattices with all other parameters fixed, and then see what happens at fixed quark mass when we decrease the size of the splitting, $\Delta_{A}$. This would correspond to staggered actions that have smaller discretization errors than the Asqtad action, such as HYP staggered fermions [20, 21].

Figures 4 and 5 show the critical $\delta_{A}^{\prime}$, Eq. (40), on the coarse and fine lattices, respectively, as a function of light quark mass at fixed strange quark mass. In lattice units, the strange quark mass is 0.05(0.031) on the coarse (fine) lattice. As noted in Ref. [6], these values are slightly larger than the physical strange quark mass, but there is no qualitative difference in the results here when we lower $a m_{s}$ by $\approx 20 \%$. The horizontal line shows the value of $\delta_{A}^{\prime}$ which was determined in chiral fits to the mass and decay constant of the pseudoscalar-taste mesons [16, 17]. We can see that we are far outside the broken phase here, for both the coarse and the fine lattices, even as we take the chiral limit. The relevant points in the parameter space are when $a m_{l} \geq 0.005(0.0062)$, since this is the range of values simulated currently on the coarse (fine) MILC lattices, and $a m_{l} \approx 0.002(0.001)$, the physical value of the average up and down quark masses on the coarse (fine) MILC lattices. ${ }^{4}$

We move closer to the broken phase when all three quark masses are light: the 3-flavor case in the chiral limit. In this case, $\delta_{c}^{\prime}=-4 \Delta_{A} / 3$, which is surprisingly close to the value of $\delta_{A}^{\prime}$ currently determined from simulations [11]. Figures [6] and [7 shows $\delta_{c}^{\prime}$ as a function of the strange quark mass at $m_{l}=0$ for the coarse and fine lattices respectively. The solid line shows the central value of $\delta_{A}^{\prime}$ that has been determined, while the dotted lines give the

\footnotetext{
4 These values for the physical light quark mass are found relative to the "nominal" strange quark mass, $a m_{s}=0.05(0.031)$ on the coarse(fine) lattices, using the ratio $m_{s} / m_{l} \approx 27[22]$.
} 
statistical error. We can see that there is a better chance to enter the broken phase for very light strange quark mass, although the error is large enough that this may not occur. A more precise determination of the hairpin parameter would allow for a concrete statement about the broken phase at light quark masses.

As is apparent from Figs. [6 and 7] as a becomes smaller, we are somewhat less likely to be in the broken phase. This seems to imply that as $a \rightarrow 0$, there is little chance we will be in the broken phase, even close to the chiral limit. This should be expected, as this phase is a lattice artifact and thus vanishes in the continuum limit (note that as $a \rightarrow 0$, $\left.\delta_{c}^{\prime} \rightarrow-\infty\right)$. This also makes it clear that at larger lattice spacing, $\delta_{A}^{\prime}$ is more likely to be in this phase. We anticipated this, since the broken phase appears when the discretization errors are comparable to the lattice quark masses. This implies that we must not simulate at too coarse a lattice spacing, and the values used here are sufficiently fine so as to not approach the broken phase. At these lattice spacings, it is clear that there is only a problem if all three quarks are very near zero, which is not a physical point in the parameter space.

One may wonder how this changes with more improved actions, such as HYP staggered fermions [20]. Let us define $\tilde{\Delta}_{A}$ as the axial-taste splitting for some more-improved action, and we define the quantity

$$
x \equiv \frac{\tilde{\Delta}_{A}}{\Delta_{A}}
$$

with $\Delta_{A}$ the Asqtad splitting. We plot in Fig. 8 for the coarse lattice spacing $a^{4} \delta_{c}^{\prime}$ as a function of $x$. We only plot the coarse lattice here, since we expect this to be the place where the problem would be more severe. These are plotted with the lightest light quark mass from the MILC simulations $\left(m_{l} / m_{s}=0.005 / 0.05\right)$. As $x$ is reduced, we see for both the coarse and fine lattices that $\delta_{c}^{\prime}$ is also reduced in magnitude. For reference, HYP fermions lie roughly at $x \approx 0.5[21]$. Whether or not $\left|\delta_{A}^{\prime}\right|$ is reduced by the same magnitude as $\Delta_{A}$ is uncertain, and this is something that must be checked in a dynamical simulation. A good improvement program should improve all the lattice artifacts to some degree, so we would expect $\left|\delta_{A}^{\prime}\right|$ to be reduced as well. As can be see from the figure, as long as $\left|\delta_{A}^{\prime}\right|$ does not increase by a significant factor after improvement (the central value for $\delta_{A}^{\prime}$ in the Asqtad case is shown by the solid line), then we will not be in the broken phase. 


\section{CONCLUSION}

We have discussed the phase structure of a lattice theory with staggered quarks, which has an approximate [to $\left.\mathcal{O}\left(a^{2}\right)\right] S O(4)$ taste symmetry, as seen in the chiral theory. This taste symmetry can be spontaneously broken down to $S O(3)$ for certain values of the parameters in the theory. This most likely will not occur for current staggered actions, even among improved theories, unless all three quark masses are close to the chiral limit. At that point, there could be a phase transition with 3 pseudo-Goldstone bosons [with masses $\sim \mathcal{O}\left(a^{4}\right)$ ], and a rather different mass spectrum than in the unbroken phase.

The possibility of a flavor-symmetry (or in this case taste-symmetry) breaking phase is a general feature of any lattice theory which has additional terms in the squared meson masses at finite lattice spacing. While originally discussed in the context of Wilson fermions (Aoki phase), we see that there are complicated ways for the taste symmetry with staggered fermions to be broken as well. The difference here is mainly that the taste symmetry is already broken by the lattice spacing, and it is only the approximate $S O(4)$ symmetry which is broken. So unlike the Wilson case, there are significant corrections at higher order in the lattice spacing.

This brings up an interesting question about the $\mathcal{O}\left(a^{4}\right)$ corrections. We are stating here that when the lattice spacing becomes too large, we are most likely to enter this broken phase. However, as $a$ increases, higher-order scaling violations become more important. It is unclear whether or not the broken phase will remain at extremely coarse lattice spacing. Using a chiral Lagrangian that includes higher order corrections [23], this could be studied by determining the relative sizes of higher-order contributions.

An interesting question arises relating to the quenched approximation of staggered fermions. For Wilson fermions, there is the possibility of entering the Aoki phase, and this can cause significant problems with locality when simulating Wilson, Domain Wall, or Overlap quarks 24]. For the staggered case, however, there is no Aoki phase in this context. There cannot be an Aoki phase in the context of the current analysis, since the $\delta_{A}^{\prime}$ splittings do not add to the mass of the $\eta_{A}^{\prime}$. So unlike the case of Wilson quarks, there is no difficulty when simulating staggered quarks in the quenched approximation.

\section{ACKNOWLEDGMENTS}


We are grateful to M. Alford, C. Bernard, M.C. Ogilvie and S. Sharpe for helpful discussions, and especially C. Bernard for helpful comments on the manuscript. This work was partially supported by the U.S. Department of Energy, under grant number DE-FG0291ER40628.

[1] C. Bernard et al., Nucl. Phys. Proc. Suppl. 106, 199 (2002).

[2] C. Bernard et al., Nucl. Phys. Proc. Suppl. 119, 257 (2003) arXiv:hep-lat/0208041.

[3] C. W. Bernard et al. [MILC Collaboration], Phys. Rev. D 61, 111502 (2000) arXiv:hep-lat/9912018.

[4] C. W. Bernard et al., Phys. Rev. D 62, 034503 (2000) arXiv:hep-lat/0002028.

[5] C. W. Bernard et al., Phys. Rev. D 64, 054506 (2001) arXiv:hep-lat/0104002.

[6] C. Aubin et al., arXiv:hep-lat/0402030.

[7] M. F. L. Golterman, Nucl. Phys. B 273, 663 (1986).

[8] W. J. Lee and S. R. Sharpe, Phys. Rev. D 60, 114503 (1999) arXiv:hep-lat/9905023.

[9] S. Aoki, Phys. Rev. D 30, 2653 (1984).

[10] C. Aubin and C. Bernard, Nucl. Phys. Proc. Suppl. 129, 182 (2004) arXiv:hep-lat/0308036.

[11] C. Aubin and C. Bernard, Phys. Rev. D 68, 034014 (2003) arXiv:hep-lat/0304014.

[12] S. R. Sharpe and R. L. . Singleton, Nucl. Phys. Proc. Suppl. 73, 234 (1999) arXiv:hep-lat/9808047.

[13] S. R. Sharpe and R. J. Singleton, Phys. Rev. D 58, 074501 (1998) arXiv:hep-lat/9804028.

[14] G. Munster, arXiv:hep-lat/0407006.

[15] S. R. Sharpe and J. M. S. Wu, arXiv:hep-lat/0407025.

[16] C. Aubin et al. [MILC Collaboration], Nucl. Phys. Proc. Suppl. 129, 227 (2004) arXiv:hep-lat/0309088.

[17] C. Aubin et al. [MILC Collaboration], arXiv:hep-lat/0407028

[18] C. Bernard [MILC Collaboration], Phys. Rev. D 65, 054031 (2002) arXiv:hep-lat/0111051.

[19] C. Aubin and C. Bernard, Phys. Rev. D 68, 074011 (2003) arXiv:hep-lat/0306026.

[20] A. Hasenfratz and F. Knechtli, Phys. Rev. D 64, 034504 (2001) arXiv:hep-lat/0103029.

[21] A. Hasenfratz, Nucl. Phys. Proc. Suppl. 119, 131 (2003) arXiv:hep-lat/0211007.

[22] C. Aubin et al. [HPQCD Collaboration], arXiv:hep-lat/0405022. 
[23] S. R. Sharpe and R. S. Van de Water, arXiv:hep-lat/0409018,

[24] M. Golterman and Y. Shamir, Phys. Rev. D 68, 074501 (2003) arXiv:hep-lat/0306002. 


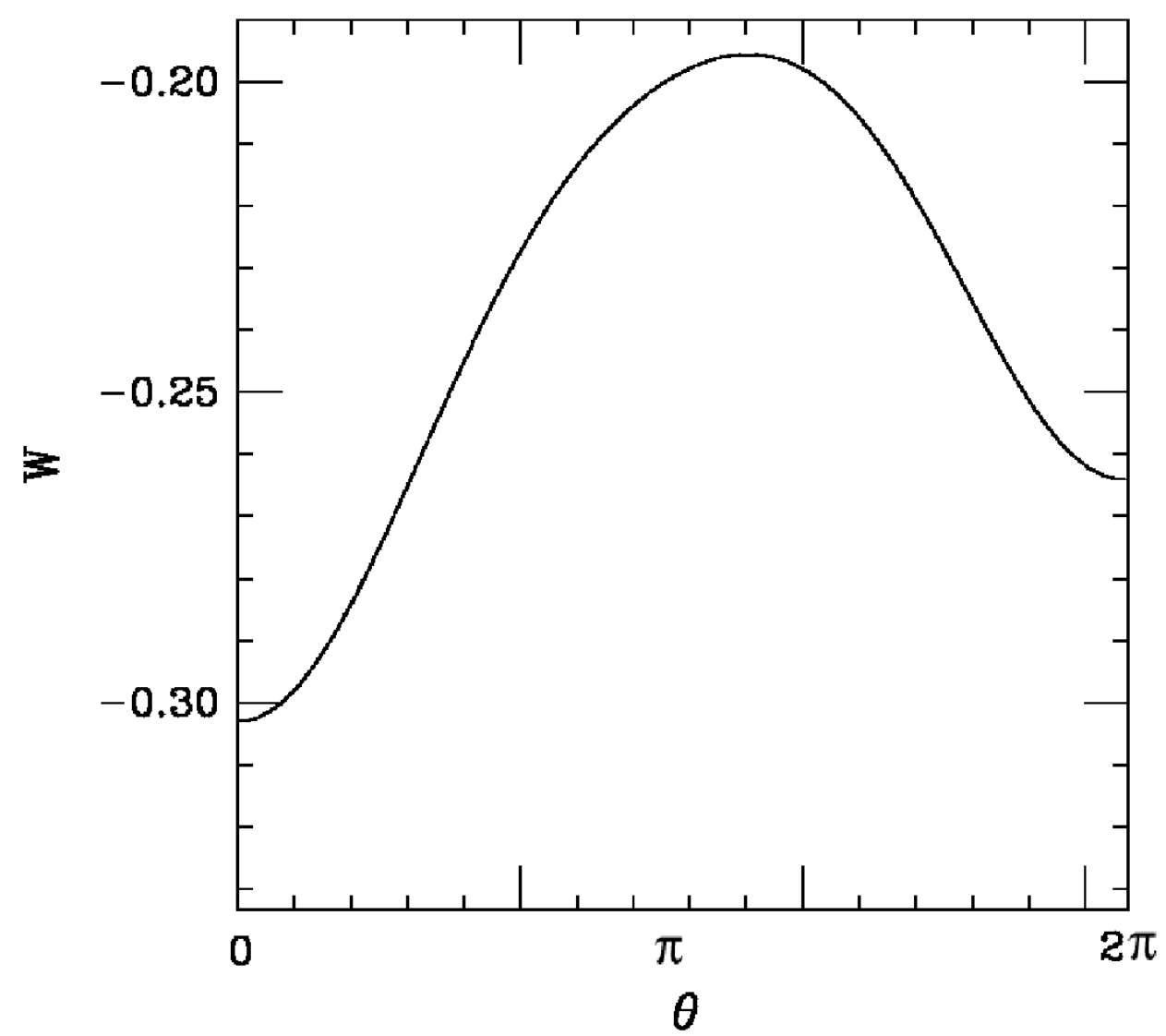

FIG. 1: Potential in the unbroken phase; parameters here are for the coarse MILC lattice, with the light/strange lattice quark masses $0.005 / 0.05$. The global minimum is clearly at $\theta=0$. 


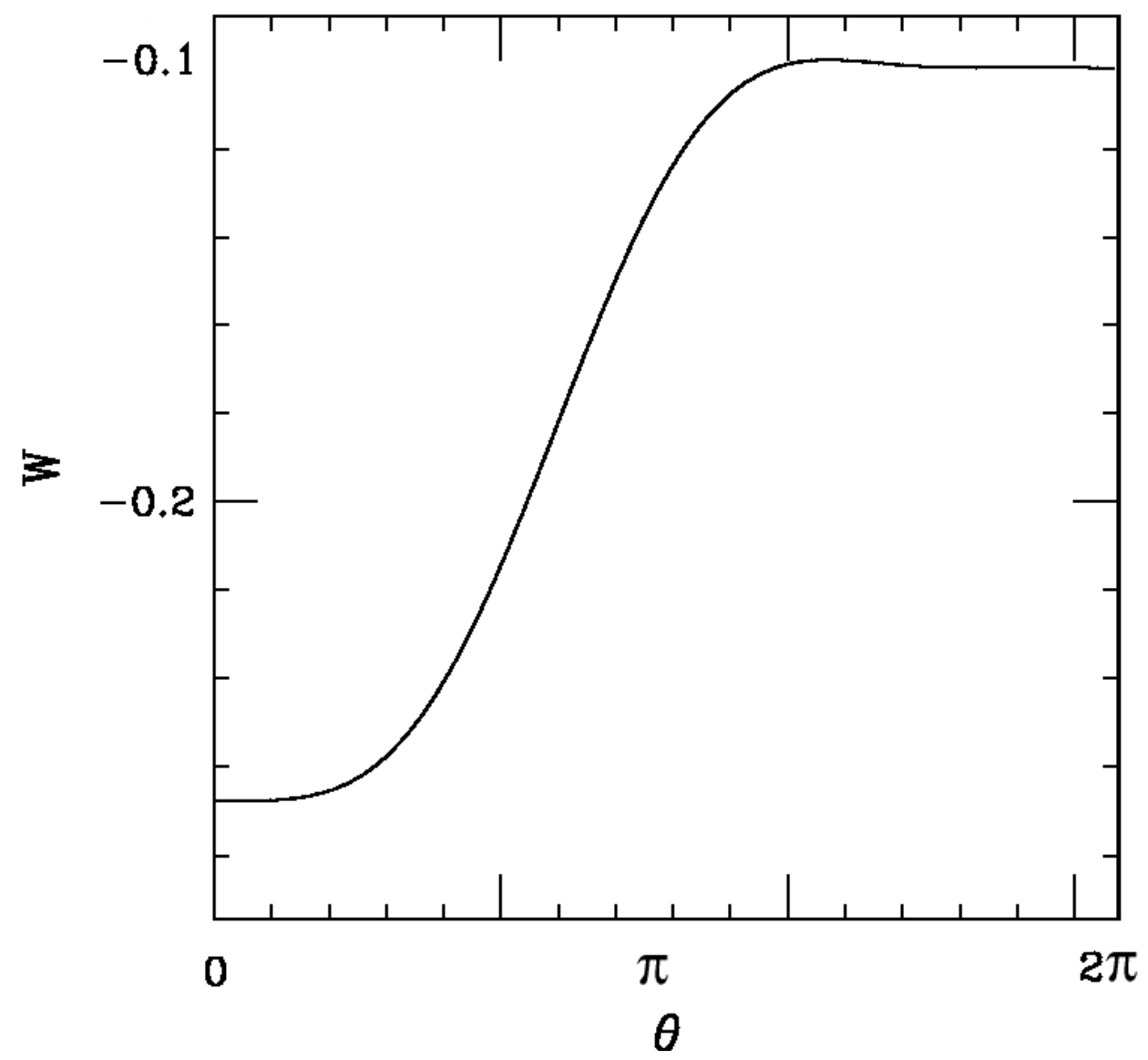

FIG. 2: The potential at the critical point, for the same masses as in Fig. 11 The region near $\theta=0$ has become flatter, as expected near a critical point. 


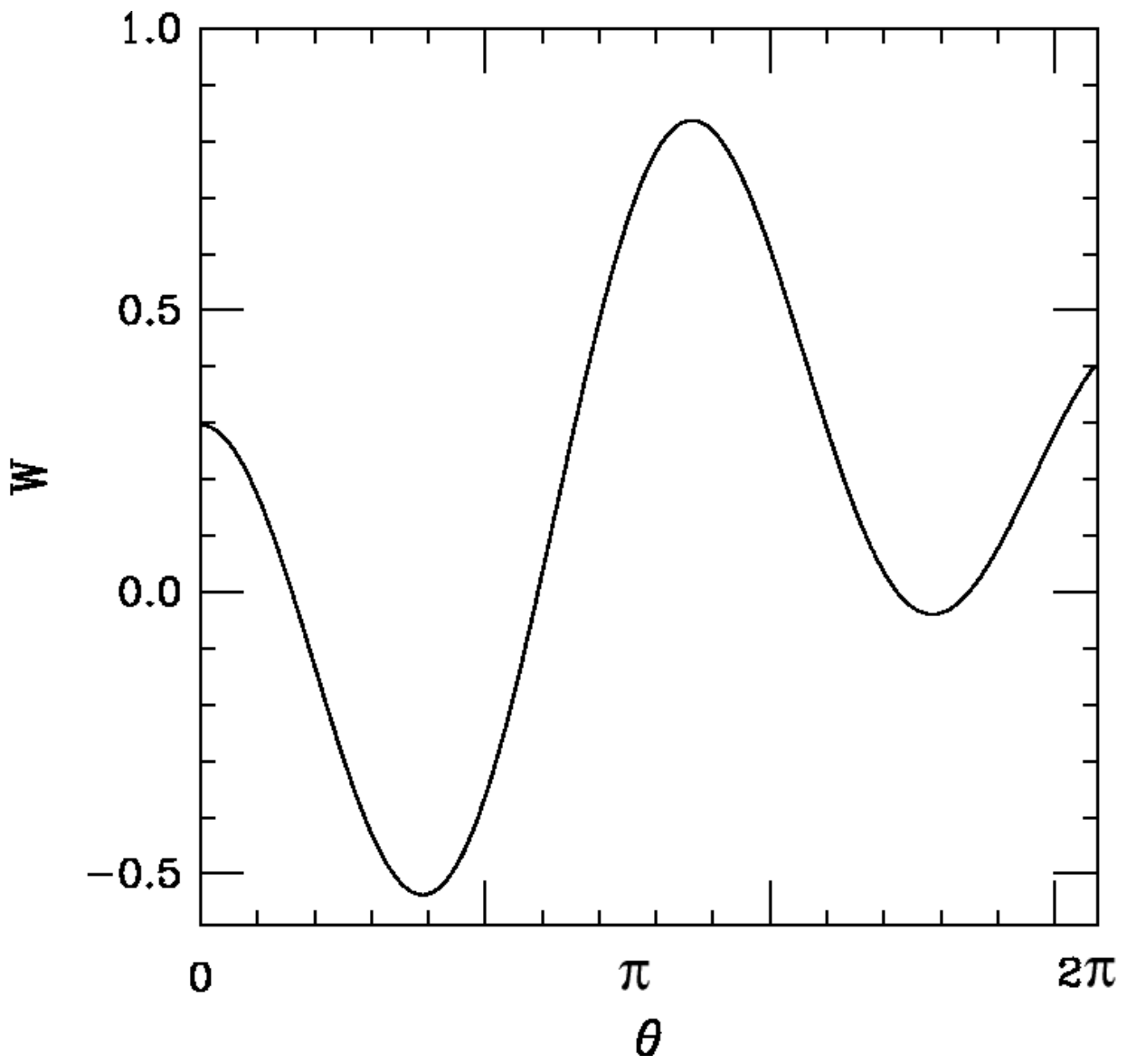

FIG. 3: The potential in the broken phase, where the global minimum now is at a non-zero value of $\theta$. Note the other (local) minimum has shifted down and to the left; as $\delta_{A}^{\prime} \rightarrow-\infty$, the two minima will both be global minima at different values of $\theta$. 


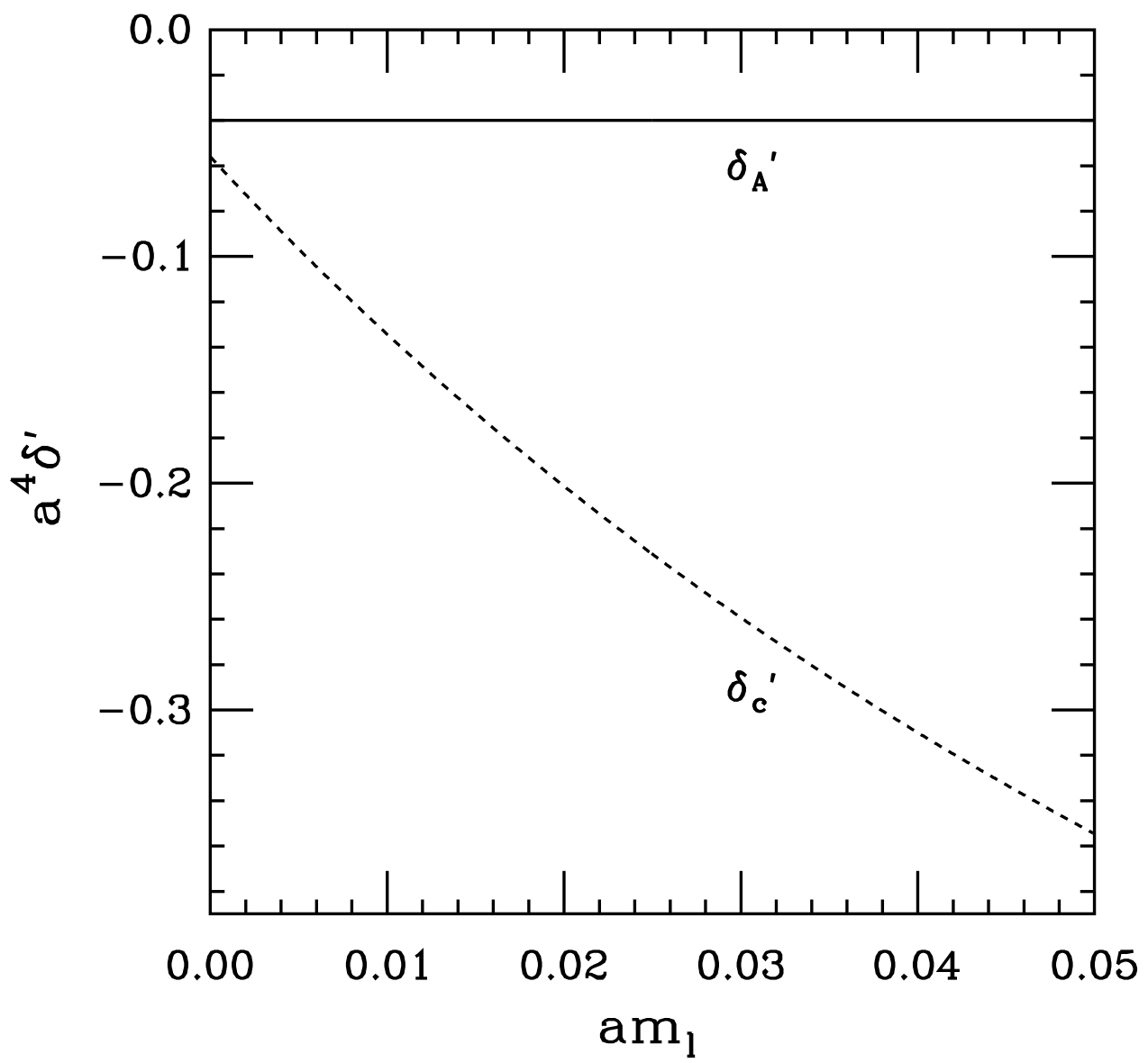

FIG. 4: The dashed line shows $\delta_{c}^{\prime}$ as a function of the light quark mass (in lattice units) on the coarse lattice, for a lattice strange quark mass of 0.05 . The solid horizontal line is the value of $\delta_{A}^{\prime}$, determined from chiral fits to the $2+1$ MILC data [16, 17]. 


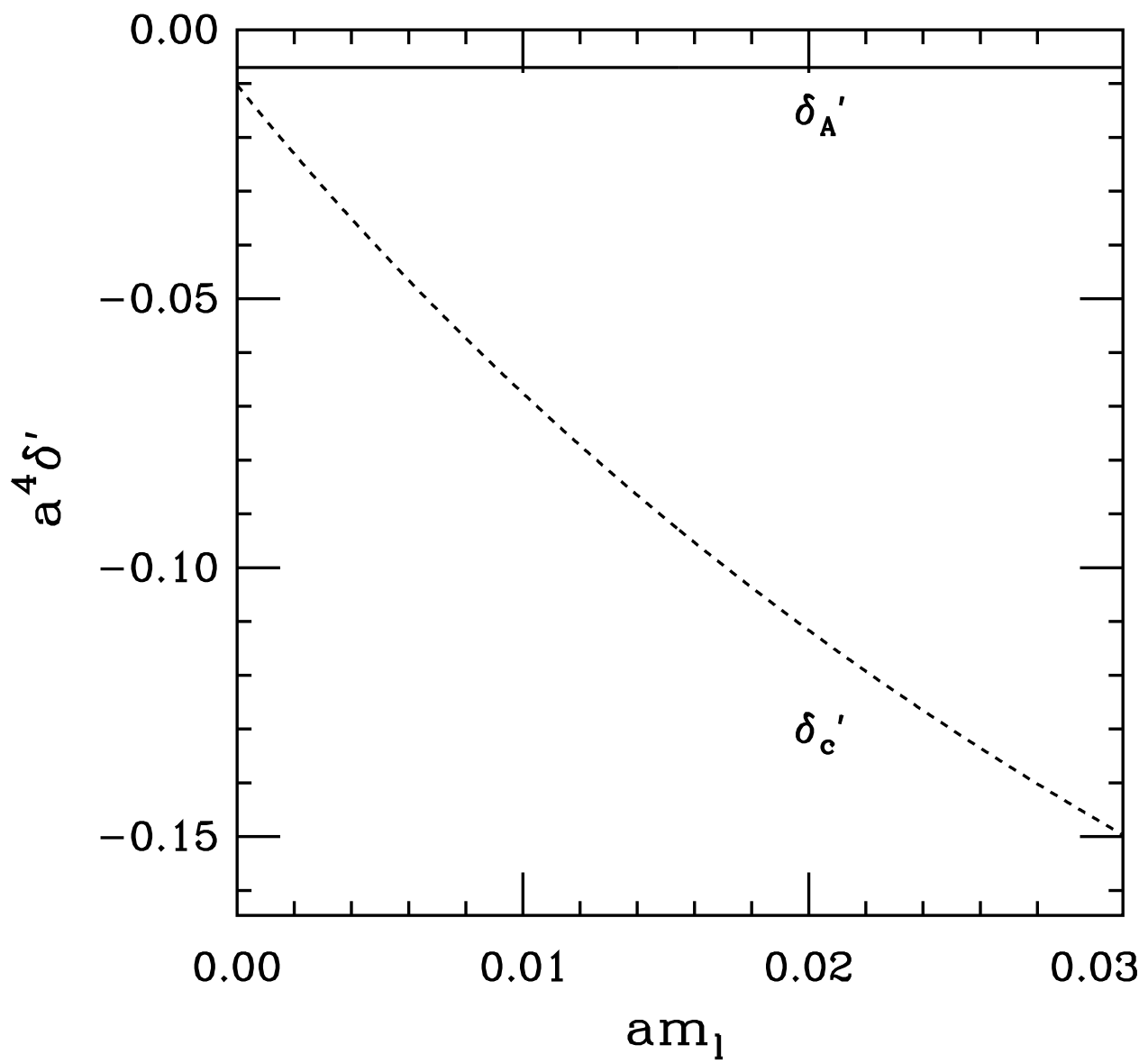

FIG. 5: Same as Fig. 4 but for the fine lattice spacing, and for a lattice strange quark mass of 0.031 . 


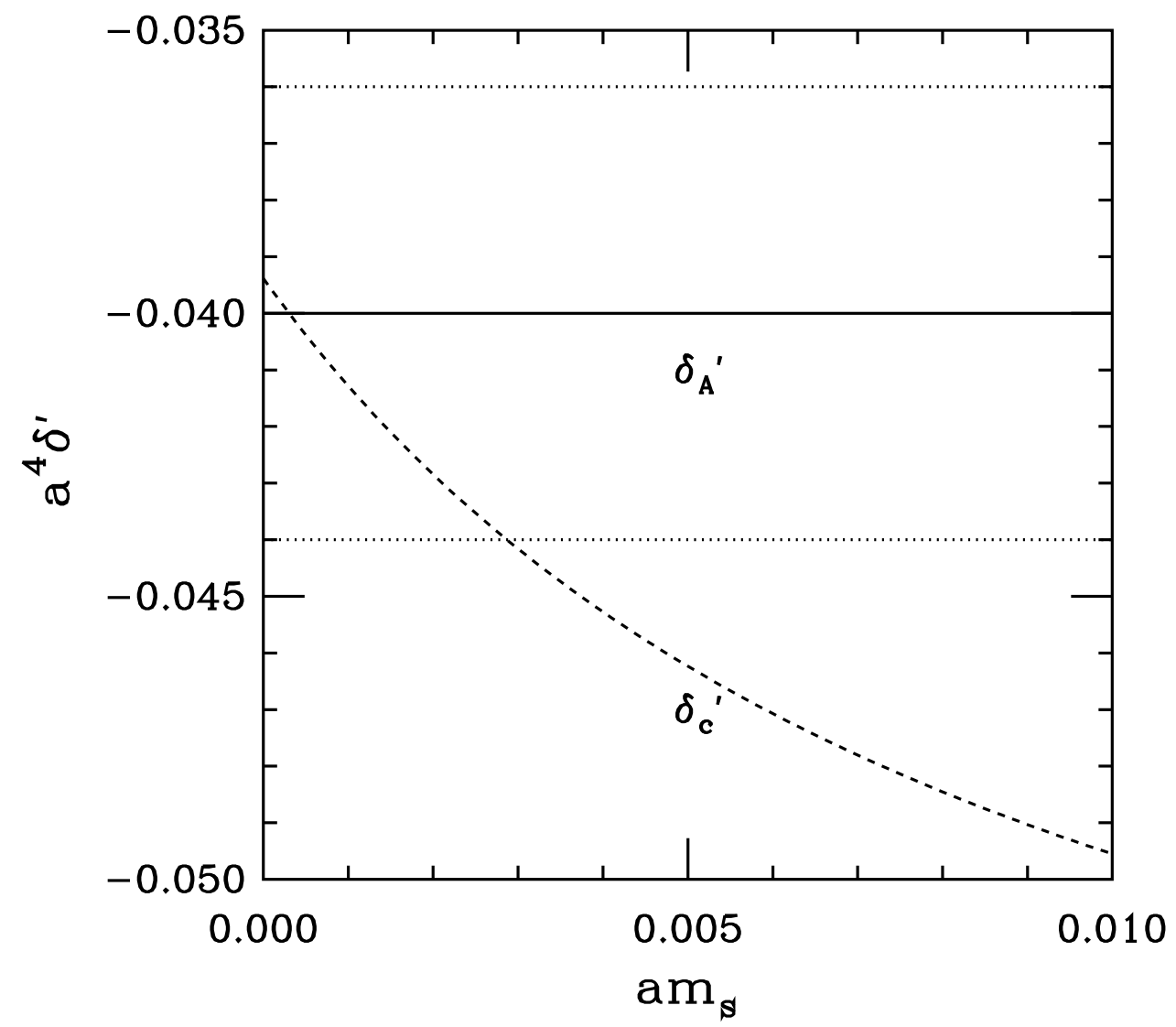

FIG. 6: The dashed line is $\delta_{c}^{\prime}$ on the coarse lattice as a function of the strange quark mass for $m_{l}=0$. The solid line is the value of $\delta_{A}^{\prime}$, with the statistical errors given by the dotted lines. For very light $m_{s}$ we see that there is a significant probability that we will enter the broken phase. 


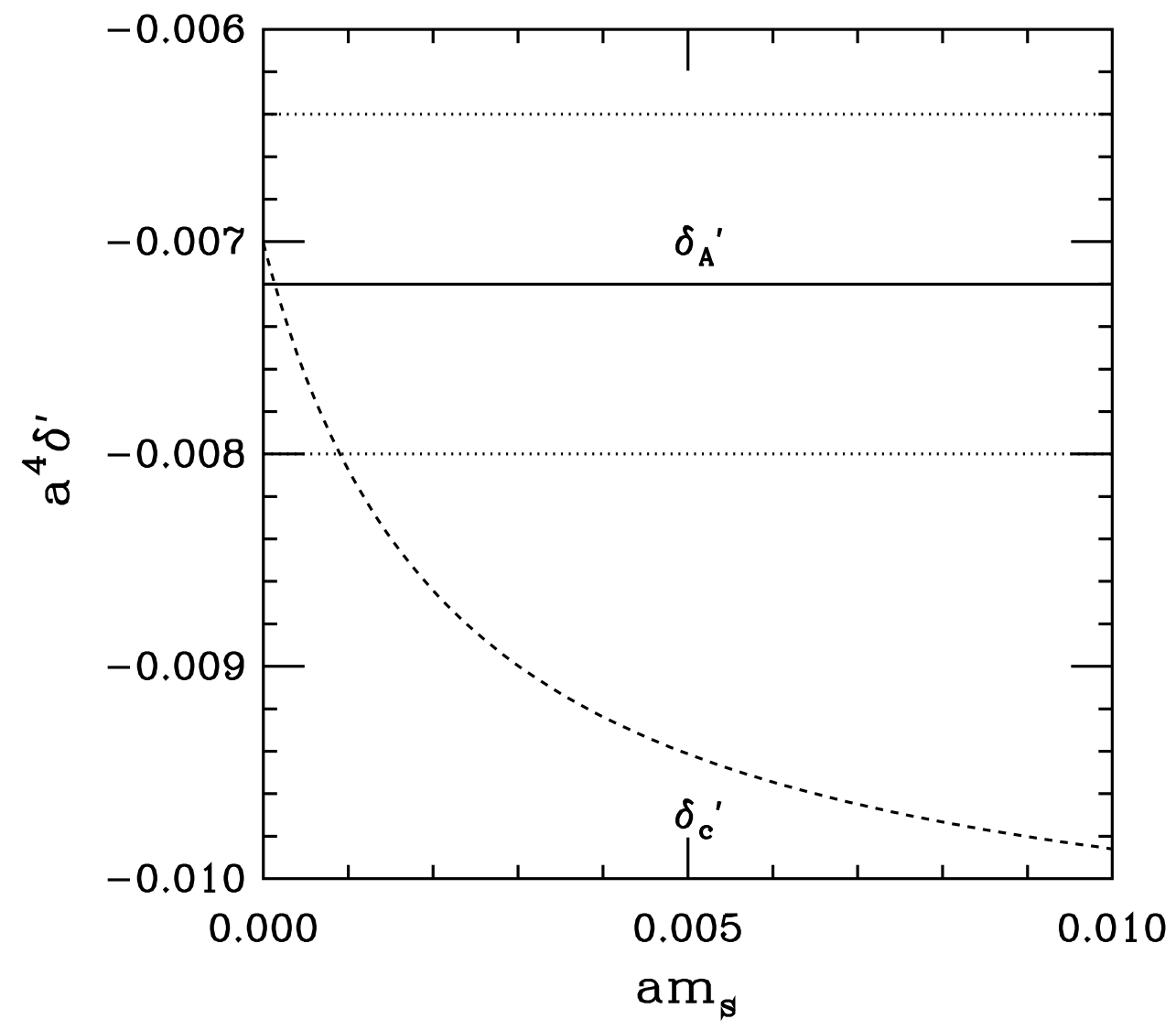

FIG. 7: Same as Fig. [6 but for the fine lattice spacing. There is still a significant probability that we could be in the broken phase for light $m_{s}$ but it is somewhat less likely than on the coarse latice. 


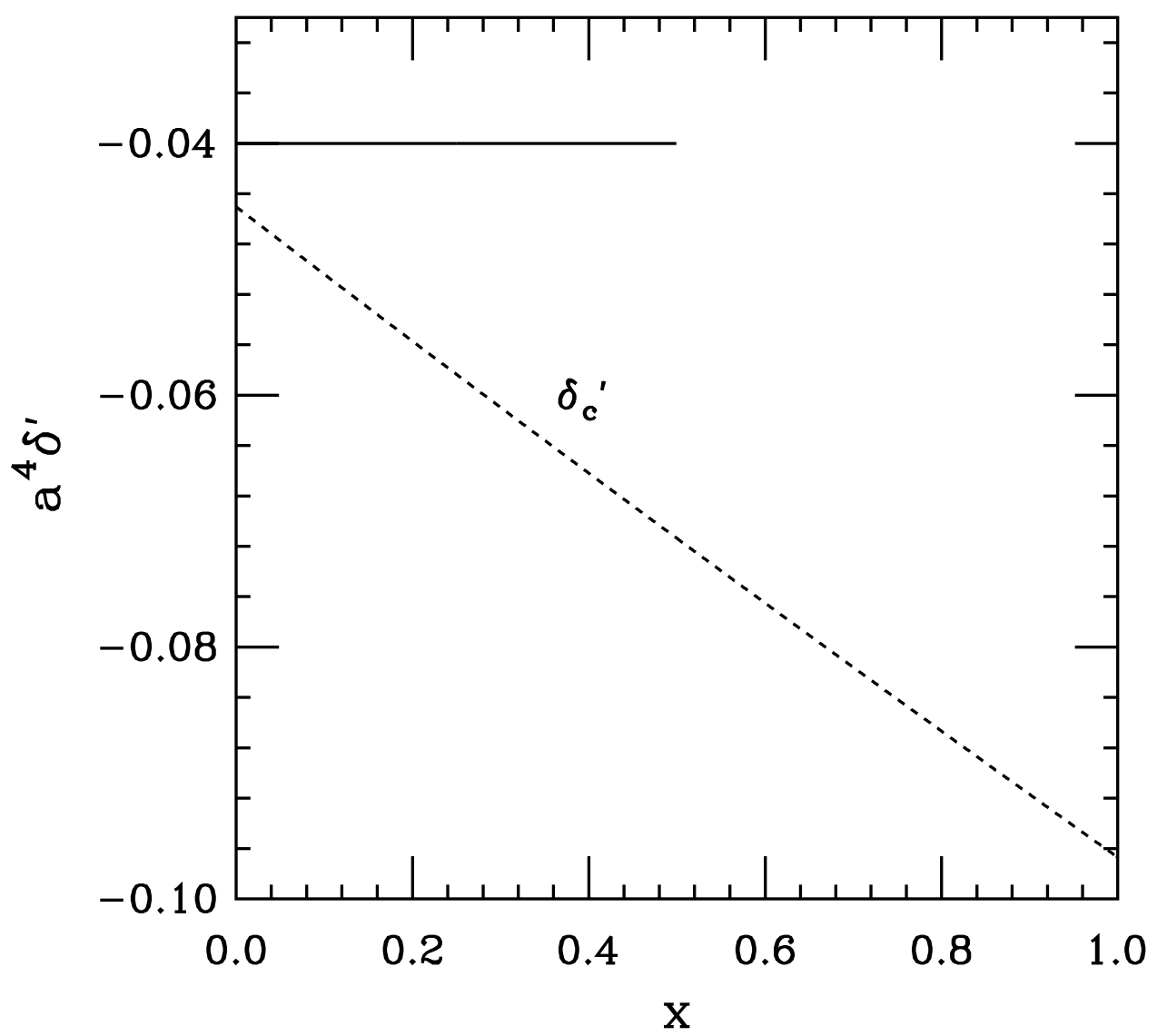

FIG. 8: $\delta_{c}^{\prime}$ versus the fractional improvement of the splitting $\Delta_{A}$ for the coarse lattice. $x=1$ corresponds to the Asqtad action, while $x \approx 0.5$ corresponds to HYP staggered fermions [20, 21]. The horizontal solid line is the value of $\delta_{A}^{\prime}$ for the Asqtad action for reference. 